\title{
Short-term recognition memory of tones
}

\section{STANLEY M. MOSS, ${ }^{2}$ JEROME L. MYERS, AND THOMAS FILMORE}

UNIVERSITY OF MASSACHUSETTS

Four Os participated in a recognition memory experiment for a period of 5 weeks. The task required $O$ to judge whether two temporally sequenced tones (ISI $=0.5,2.0$, or $8.0 \mathrm{sec})$ were the "same" or "different." Latencies and confidence ratings were obtained for each judgment. TSD analyses applied to individual $O$ 's data indicated consistent and rapidly decreasing $d^{\prime}$ s as a function of ISI. ROC functions generated from the latencies and ratings produced comparable results, indicating the feasibility of using latency measures along with the type of judgments made to obtain sensitivity measures. Response bias, as indicated by the differences in the latencies between "same" and "different" judgments, did not produce consistent trends.

Several recent experiments have been concerned with recognition memory for the pitch of pure tones. In these experiments, a trial consisted of the presentation of a standard tone followed some time later by a comparison tone; the $O$ was required to determine whether the two tones had the same or different pitches. Two issues have arisen in this research: (1) the nature of the relationship between recognition memory and the interval between the two stimuli (the interstimulus interval, ISI), and (2) the relative response times on the two types of trials, those on which standard and comparison were the same ( $S$ trials) and those on which they were different (D trials). The present study was designed to investigate these issues further.

ISI effects. Consistent with the obvious expectation, Bindra, Williams, and Wise (1965) found a higher error rate for an ISI of $10 \mathrm{sec}$ than for an ISI of $1 \mathrm{sec}$. However, the use of only two points precludes any judgments about the shape of the function relating memory to ISI. Wickelgren (1966), who used ISIs of 2,4 , and $8 \mathrm{sec}$ with a tone filling the intervals, found that strength of memory declined exponentially with increased ISI. The measure of memory strength was $d^{\prime}$, a construct derived from the theory of signal detection (Swets, Tanner, \& Birdsall, 1961), which has the advantage of being independent of response bias. Massaro (1968), who used filled intervals of 1,2 , and $4 \mathrm{sec}$, also obtained an exponential decline in ' $d^{\prime}$ with increased ISI. However, the rate of decline was very small when the interval was blank. Aiken and Lau (1966) obtained even less of an effect of ISI using blank intervals of $.95,4.5$, and 8.9 sec duration. Values of 'd' estimated from the data presented in their article are almost invariant over levels of ISI.

The failure of Aiken and Lau to obtain an ISI effect may be due to their use of a single standard tone. Harris (1952) has pointed out that such a procedure may enhance long-term memory for the standard, retarding forgetting. However, Massaro (1968) employed three randomly sequenced standard tones and, under the blank interval procedure, found a low rate of decline in $d^{\prime}$ over intervals. Either these results are due to other, undetected methodological flaws or, as Melton (1963) has suggested, decay is not a factor in memory, or the bulk of the decay occurs within the first second following the offset of the standard tone. In order to investigate the last hypothesis, the present study included an ISI of $.5 \mathrm{sec}$ along with ISIs of 2 and $8 \mathrm{sec}$.

Response times on $S$ and $D$ trials. Bindra and his associates (Bindra, Williams, \& Wise, 1965; Bindra, Donderi, \& Nishisato, 1968 ), using pure tones and $S$ and $D$ trials of equal frequency, found that the proportion of "different" responses exceeded the proportion of "same" responses, and that response latencies were longer on $S$ than on D trials. In a related study, Coltheart and Curthoys (1968) found that increases in the proportion of $S$ trials were accompanied by an increase in "same" responses and by a reduction in the latency discrepancy between S and D trials; in fact, with $70 \%$ and $90 \% \mathrm{~S}$ trials, latencies were longer on $D$ trials. The relation between response bias and the difference in response times for $S$ and $D$ trials appears well establsihed; the effects of discriminability are less clear. Bindra et al (1968) have taken the position that increased difficulty does not necessarily make the latencies of "same" responses increasingly longer than those of "different" responses. In addition, they feel that practice does not influence the difference in latencies. The response time data of the present study, obtained under three ISIs and over a period of extended practice, aid in clarifying these points.

A second reason for collecting latency data was to enable a comparison of ROC curves based on rating and latency data. Latencies can be viewed as points along a continuous rating scale, and ROC curves can then be derived in the manner described by Green and Swets (1966). Norman and Wickelgren (1969) constructed ROC curves in this manner, using visually presented digits, and obtained functions that were peaked at the "yes-no" point. We were interested in determining whether the functions reported by Norman and Wickelgren would be observed in the present study and whether they would be affected by ISI.

\section{METHOD}

\section{Observers}

Four right-handed undergraduate males were used in the present experiment. All reported no known hearing losses. They all received $\$ 1.50$ per experimental session and an additional $\$ 5.00$ bonus for completing the entire experiment.

\section{Apparatus}

The response console was a $12 \times 20$ in. black sloped panel placed on a 27 -in.-high table. In the lower left-hand corner of the panel, parallel to the front edge, were three black rectangular pushbutton switches covering an area of $2 \times 4 \mathrm{in}$. The center switch was 1 in. wide and each side switch was $1 \frac{1}{2} \mathrm{in}$. wide. The tops of these switches were raised $1 / 4$ in. above the surface of the panel. Typed labels, "same" and "different," were pasted on the panel surface immediately above the left and right switches, respectively. Six inches to the right of the center button was a vertical array of five $1 / 2$-in. white plastic-capped pushbutton switches. The centers of these were 1 in. apart, with the bottom-most switch lined up with the centers of the rectangular buttons. Immediately to the right of the bottom and top buttons were the typed labels "not confident at all" and "very confident," respectively. Twelve inches up from the bottom edge of the panel was a $21 / 2$-in. vertical panel in which was mounted a $1 / 2$-in.-diam red-jeweled incandescent lamp. A 3-in. permanent magnet speaker was mounted at the surface of a 63/4-in. panel extending beyond the top of the vertical panel.

The stimuli were tones generated by and gated through a BRS solid-state system to the speaker. The sequence of stimuli and 
interstimulus intervals (ISI) were programmed on eight-channel punched paper tape. O's responses and latencies were automatically recorded on a Digitec printer at the completion of each trial.

\section{Procedure}

Six 70-dB (SPL) tones and three ISI (.5, 2 , and $8 \mathrm{sec}$ ) were used in the present study. The tones were divided into two nonoverlapping subsets: $1,000,1,010$, and $1,020 \mathrm{~Hz}\left(S_{1}, S_{1}\right.$, and $\left.S_{1+}\right)$ and 1,030 , $1,040,1,050 \mathrm{~Hz}\left(S_{2-}, S_{2}\right.$, and $\left.S_{2+}\right)$. The sequence of events during a trial consisted of the following: a .5-sec flash of the red-jeweled lamp (waming signal), a 2-sec foreperiod interval; a $.5-\mathrm{sec}$ duration standard tone $\left(S_{1}\right.$ or $\left.S_{2}\right)$, an ISI, and finally, a .5-sec duration tone from the same subset as the standard just presented.

The Os were instructed to depress the center rectangular button with the index finger of their right hands when the warning signal occurred. After they heard the second tone and had made their decision that this tone was the "same as" or "different from" the first tone, they were to respond by removing their index fingers and depressing the appropriate side button. After they had made this decision, they were instructed to rate it by depressing one of the five buttons to the right, indicating their degree of confidence.

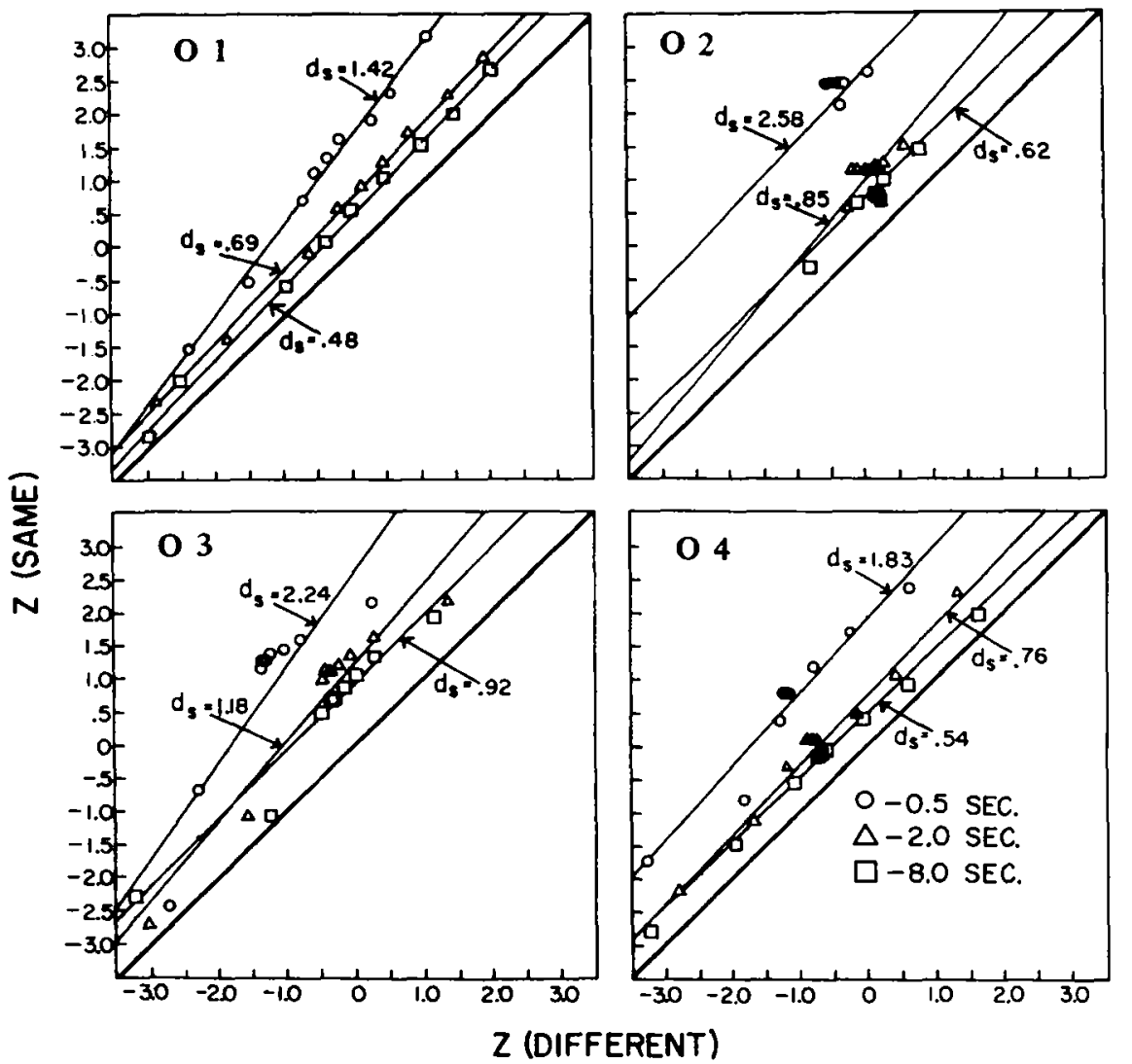

Latencies were measured as the lapsed time between the onset of the second tone and the decision response. The average intertrial interval was $7 \mathrm{sec}$; this included the decision latency, the time required to rate that decision, and a fixed 5-sec interval between the rating response and the onset of the next trial warning signal.

Nine random orders of 64 trials were placed on punched paper tape, three for each ISI. Each subset of tones was represented with equal frequency in each random order. Within each subset, the second tone was the same as the first on 16 trials, either an $S_{1}$ - or an $S_{2}$ on 8 trials or an $S_{1}+$ or an $S_{2}+$ tone on 8 trials. Thus, the proportion of same trials for each random order was .50. Each $\mathrm{O}$ was presented, in random order, one of the three tapes for each ISI during a session, with the restriction that all tapes be used with equal frequency during a span of nine sessions.

All Os participated for 28 consecutive 1 th weekday sessions, with the first three sessions considered as practice.

\section{RESULTS AND DISCUSSION \\ Choice and Rating Data}

The memorial process. Several papers have indicated that memory for a variety of verbal and physical stimuli can be treated adequately within the framework w TSD. Following Wickelgren (1969), we assume that the same-different judgment is based on the similarity of the comparison tone with the standard tone, that similarity is a normally distributed random variable. and that the $O$ responds "same" if, and only if, familiarity exceeds $X_{C}$, the criterion for a same response. This approach can readily be extended to confidence ratings (Green \& Swets, 1966). In the present experiment, the 10 responses obtained from the combination of the same-different response and the 5 -point rating scale can be viewed as lying on a continuum from "5-same" to "5-different." A "5-same" response implies that the comparison tone was very familiar, a "5-different" response implies very weak familiarity, and the other response classes imply intermediate values of familiarity. We can view the $O$ as having nine different criteria that give rise to nine different probabilities of "same" responses; the proportion of "5-same" responses is the probability that familiarity exceeds the most familiar cutoff, the proportion of "4-same" or "5-same" responses is the probability that the next most familiar cutoff is exceeded, and so on. Treating the probabilities on $S$ and $D$ trials separately, we can obtain nine pairs of hit and false-alarm probabilities. (Note that a "hit" is defined throughout this paper as a "same" response on $S$ trials; a false alarm is a "same" response on D trials.)

The procedure just described was carried out by pooling probabilities over the 5 weeks and two standard stimuli $\left(S_{1}\right.$ and $S_{2}$ ). While the data showed some variability over the weeks. of the experiment, no systematic trend was evident; nor did the two stimuli have any clear effect upon responding. The obtained proportions are plotted on normal-normal paper in Fig. 1. The slopes tend to be close to, but slightly greater than, 1.0, indicating a slightly greater variance of comparison tone familiarity on D than on $S$ trials, a result consistent with previous findings by Wickelgren (1966). The slopes decrease monotonically, approaching unity, with increased ISI, indicatıng more equitable use of the rating scale on $S$ and on D trials. One exception to this is seen in $\mathrm{O} 2$, who used only a portion of the rating scale. While $d^{\prime}$, distance from the positive diagonal along the negative diagonal,

Fig. 1. ROC functions for $01-04$ obtained from all of the rating data under each ISI. The $d_{s}$ values were calculated as the distance along the main negative diagonal between the main . positive dingonal and each ROC function (Egan \& Clarke, 1966). 


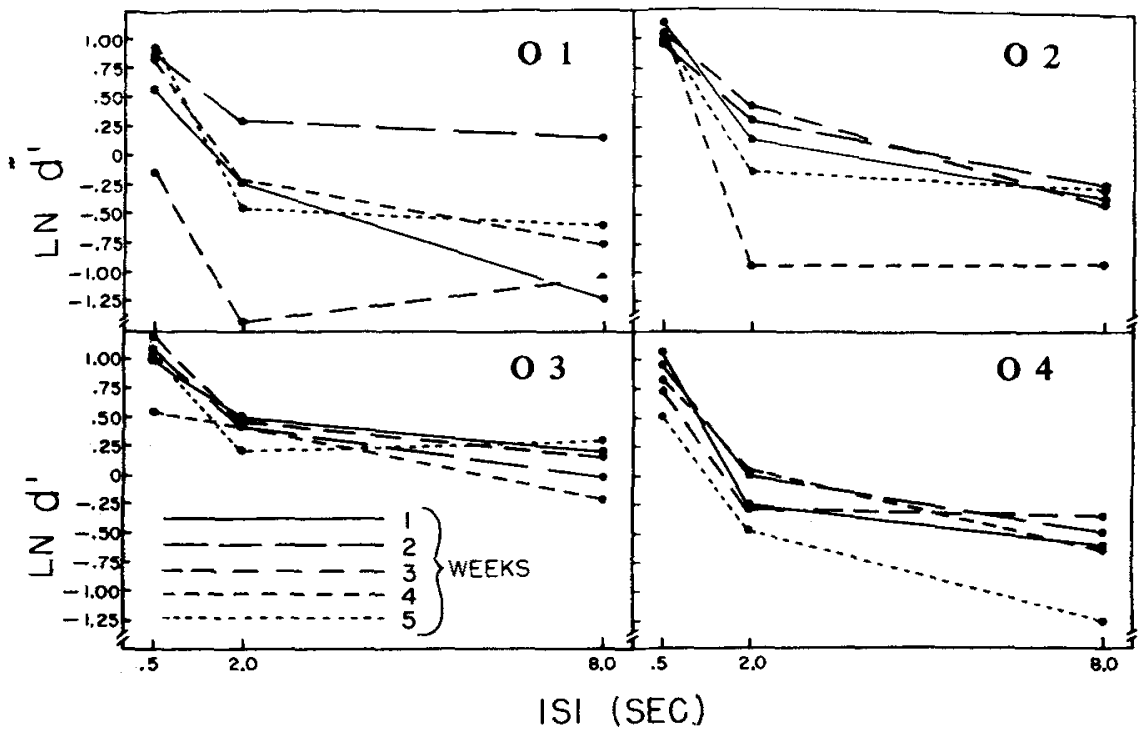

clearly decreased monotonically with increased ISI, the most marked change occurs between .5 and 2 sec. Aiken and Lau (1966) and Massaro (1968), both of whom also used blank ISI, but in the range of 1 to $10 \mathrm{sec}$, found about the same or less change in $d^{\prime}$ as was found in the present study between ISI of 2 and $8 \mathrm{sec}$. The conclusion that there is little change in memory strength over blank intervals clearly stems from the failure of these investigators to include sufficiently short intervals.

One possible interpretation of the $\mathrm{d}^{\prime}$-ISI relationship is that there are two memory traces, one a short-term trace that lasts less than $1 \mathrm{sec}$, and the other an intermediate trace that has a far slower rate of decay. Wickelgren (1969) has pointed out that, if the rate of decay of each trace is exponential, the plot of $\log d^{\prime}$ over ISIs will be linear if there is a single trace, curvilinear (concave upwards) if two traces with disparate decay rates are present. Figure 2 presents $\log d^{\prime}$ for each week for each 0 .

These results lead to an interesting speculation about the memorial process within the context of the present experiment. It was originally assumed that tones of the type used in the present experiment would not be readily encoded and therefore would enter a rapidly decaying memory store. This assumption was borne out, in part, as seen by the steeper slope component in Fig. 2. The remaining component may reflect a portion of the original stimulus trace that is encoded. In other words, the stimulus trace enters both a rapid decaying "iconic" store and a slow decaying "symbolic" store. Massaro (1968), using a similar paradigm with tones, reported a decrement in the strength of the memory trace when interpolated tones were presented, indicating the possibility of interference with the original trace. Since the ISIs that he used were relatively long, it is reasonable to assume that the interpolated tones interfered with a memory trace that was in the "symbolic" store. This interpretation is consistent with the distinction between primary memory and secondary memory made by Waugh and Norman (1965), in which only secondary memory is affected by interfering material. The consistency of the dual trace functions in the present study over the 5-week sessions is quite evident, indicating that the encoding process is established early in training.

Response bias. Thus far we have considered the effects of ISI upon the memorial process. The data obtained by Aiken and Lau (1968) suggest that ISI also affects response biases; although pitch discrimination varied little over the ISI range of .95 to $8.9 \mathrm{sec}$, the overall proportion of "different" responses increased from $39 \%$ to $56 \%$. Similar proportions calculated in the present study (Table 1) are less conclusive. Observer 4 shows the trend noted for the Aiken and Lau study, and this pattern is consistent over the 5 weeks of the experiment. Observers 1 and 2 exhibit a different pattern but, again, one that is consistent throughout the experiment; per cent

Table 1

Proportion "Different" Responses as Function of ISI and Observer

\begin{tabular}{llll}
\hline & \multicolumn{3}{c}{ Function of ISI and Observer } \\
\hline & \multicolumn{3}{c}{ Interstimulus Interval (Sec) } \\
\cline { 2 - 4 } & 0.5 & 2.0 & 8.0 \\
\hline $\mathrm{O}_{1}$ & .359 & .306 & .395 \\
$\mathrm{O}_{2}$ & .299 & .274 & .317 \\
$\mathrm{O}_{3}$ & .502 & .395 & .441 \\
$\mathrm{O}_{4}$ & .544 & .621 & .639 \\
\hline
\end{tabular}

Fig. 2. Log (base e) $d^{\prime}$ for 0 1-0 4 as a function of ISI and week of performance. These d's were calculated from the hit and false-alarm rates under each condition.

"different" decrease between .5 and 2 sec and increase again between 2 and $8 \mathrm{sec}$. The third $O$ shows no consistent pattern over the weeks of the experiment. Whether or not these results are at variance with the Aiken and Lau results is hard to determine, since the earlier study reports data pooled over six Os and differs in several procedural details from the present study. In particular, Aiken and Lau employed only a single standard and used comparison tones differing only by from 1 to $5 \mathrm{~Hz}$ from the standard. Data on the effects of discrimination difficulty from the Bindra et al (1968) study might be relevant; however, with a 2-sec ISI, per cent "different" was highest under a medium difficulty condition. Thus, no consistent picture emerges across studies or across Os within the present study, and it would appear that the effects of delay of discrimination difficulty upon response bias still remain to be clearly delineated.

\section{Response-Time Data}

Mean response times. The response-time data were partitioned into three different groups of response categories: (1) hit-false alarm-correct rejection-miss, (2) correct-incorrect, and (3) "same-different" (whether the comparison and standard tones were same or different from each other). Analyses of variance were carried out on the mean response times for each of these partitions, with subject, week, standard tone, ISI, and response category as dependent variables. Despite a lack of power due to the low numbers of error dfs, response time increased significantly over ISI in all analyses $(p<.01)$ and correct responses were significantly faster than incorrect responses $(p<.01)$. Few of the other sources of variance even approached significance. The means for each S, ISI, and response category for the $a$ and $b$ partitions are presented in Table 2.

Table 3 presents mean response times for same-different trials for individual ISIs and $O$ s. For $O 1$ and $O 2$, response times are faster when standard and comparison tones are the same, except for the 8-sec ISI of $\mathbf{0 1}$. The opposite relationship holds for 03 and $O$ 4; D times are faster. The rate of change of $L_{S}-L_{D}$ (latency on $S$ trials minus latency on $D$ trials) as a function of ISI also is consistent over Os.

Averaging over a much larger group of relatively unpracticed $O s$, Bindra et al (1968) found that mean $D$ times were faster than mean $S$ times. To account for 
Table 2

Mean Latencies as a Function of (a) Hit-False Alarm-Miss-Correct Rejection and (b) CorrectIncorrect for Each Observer and ISI

\begin{tabular}{|c|c|c|c|c|c|c|c|}
\hline & \multirow{2}{*}{$\begin{array}{c}\text { ISI } \\
\text { (Sec) }\end{array}$} & \multicolumn{4}{|c|}{ (a) } & \multicolumn{2}{|c|}{ (b) } \\
\hline & & Hits & F.A. & Misses & C.R.s & Correct & Incorrect \\
\hline Observer 1 & $\begin{array}{l}0.5 \\
2.0 \\
8.0\end{array}$ & $\begin{array}{l}1.163 \\
1.370 \\
1.527\end{array}$ & $\begin{array}{l}1.341 \\
1.470 \\
1.566\end{array}$ & $\begin{array}{l}1.489 \\
1.768 \\
1.722\end{array}$ & $\begin{array}{l}1.288 \\
1.521 \\
1.528\end{array}$ & $\begin{array}{l}1.210 \\
1.414 \\
1.527\end{array}$ & $\begin{array}{l}1.388 \\
1.535 \\
1.629\end{array}$ \\
\hline Observer 2 & $\begin{array}{l}0.5 \\
2.0 \\
8.0\end{array}$ & $\begin{array}{l}1.124 \\
1.444 \\
1.833\end{array}$ & $\begin{array}{l}1.434 \\
1.715 \\
1.960\end{array}$ & $\begin{array}{r}.972 \\
1.685 \\
2.045\end{array}$ & $\begin{array}{l}1.338 \\
1.539 \\
1.830\end{array}$ & $\begin{array}{l}1.202 \\
1.474 \\
1.825\end{array}$ & $\begin{array}{l}1.463 \\
1.709 \\
1.974\end{array}$ \\
\hline Observer 3 & $\begin{array}{l}0.5 \\
2.0 \\
8.0\end{array}$ & $\begin{array}{l}1.776 \\
2.083 \\
2.212\end{array}$ & $\begin{array}{l}2.266 \\
2.413 \\
2.424\end{array}$ & $\begin{array}{l}1.875 \\
2.214 \\
2.447\end{array}$ & $\begin{array}{l}1.398 \\
1.770 \\
1.961\end{array}$ & $\begin{array}{l}1.586 \\
1.951 \\
2.097\end{array}$ & $\begin{array}{l}2.048 \\
2.370 \\
2.432\end{array}$ \\
\hline Observer 4 & $\begin{array}{l}0.5 \\
2.0 \\
8.0\end{array}$ & $\begin{array}{l}1.585 \\
1.940 \\
2.271 \\
\end{array}$ & $\begin{array}{l}1.793 \\
2.281 \\
2.204 \\
\end{array}$ & $\begin{array}{l}1.407 \\
1.900 \\
2.054\end{array}$ & $\begin{array}{l}1.258 \\
1.676 \\
1.827\end{array}$ & $\begin{array}{l}1.410 \\
1.777 \\
2.002\end{array}$ & $\begin{array}{l}1.741 \\
2.021 \\
2.081\end{array}$ \\
\hline
\end{tabular}

this and the result that the proportion of "different" responses exceeded the proportion of "same" responses, they assumed, within the context of TSD, an 0 whose response times vary inversely with distance of the sampled familiarity trace from the cutoff, $X_{C}$. Such a model predicts a direct relationship between $L_{S}-L_{D}$ and proportion of "different responses." Data relevant to this prediction are available in Tables 1 and 3; proportion "different" is greater than .5 when $\mathrm{L}_{\mathrm{D}}<\mathrm{L}_{\mathrm{S}}$ and less than .5 when $L_{D}>L_{S}$ in 9 of 12 instances. However, the stronger prediction, i.e., that $L_{S}-L_{D}$ times vary directly as a function of proportion "different," does not hold for the present data. On the basis of this, it was decided that a further evaluation of Bindra's model within the context of the present study was unwarranted.

$R O C$ curves based on response times. Although it has rarely been done, response times can be used to generate ROC curves in much the same way that rating data are. Very fast "same" responses are viewed as reflecting strong familiarity of the comparison tone and very fast "different" responses are viewed as reflecting weak familiarity. The range from 0 to $5 \mathrm{sec}$ (5 sec was the longest latency encountered in the study) was divided into $20250-\mathrm{msec}$ intervals; a 40-point "scale" was generated by ordering the intervals from the fastest "same" interval to the fastest "different" interval. The points for the ROC curve are then obtained exactly as with the 10-point rating scale. The results are plotted on normal-normal paper in Fig. 3.

The ROC curves in Fig. 3 provide information similar to that in Fig. 1. Again,

Fig. 3. ROC functions for O 1-0 4 obtained from all of the latency data under each ISI. The $d_{s}$ values were calculated as the distance along the main negative diagonal between the main positive diagonal and each ROC function (Egan \& Clarke, 1966).
Table 3

Mean Latencies for "Same" (LS) and "Different" ( $\left.L_{D}\right)$ Responses as a Function of ISI and Observer

\begin{tabular}{rlrrr}
\hline & & \multicolumn{3}{c}{ Interstimulus Interval (Sec) } \\
\cline { 3 - 5 } $\mathrm{O}_{1}$ & & \multicolumn{1}{c}{0.5} & 2.0 & \multicolumn{1}{c}{8.0} \\
\hline & LS & 1.203 & 1.434 & 1.587 \\
& LS & 1.302 & 1.471 & 1.544 \\
& LS-LD & -0.099 & -0.037 & 0.043 \\
$\mathrm{O}_{2}$ & LS & 1.135 & 1.472 & 1.869 \\
& LD & 1.367 & 1.629 & 1.895 \\
& LS-LD & -0.232 & -0.157 & -0.026 \\
$\mathrm{O}_{3}$ & LS & 1.784 & 2.106 & 2.263 \\
& LD & 1.490 & 2.006 & 2.132 \\
& LS-LD & 0.294 & 0.100 & 0.131 \\
& LS & 1.587 & 1.906 & 2.143 \\
$\mathrm{O}_{4}$ & LD & 1.329 & 1.817 & 1.930 \\
& LS-LD & 0.258 & 0.089 & 0.213 \\
\hline
\end{tabular}

An interesting feature of Fig. 3 is the increases monotonically as the increases, with the largest change occurring between .5 and $2 \mathrm{sec}$. Again, the slopes of the ROC curves are close to 1.0 but, as in Fig. 1, slightly above. 'The only marked exceptions to this last statement are the slope for the $.5-\mathrm{sec}$ delay curve for 02 , which is rather high, and the slope for the .5 -sec delay for 04 , which is clearly below 1.0. Least-squares estimates of the slopes and slope-intercepts from Figs. 1 and 3 are presented in Table 4; they are generally in good agreement.
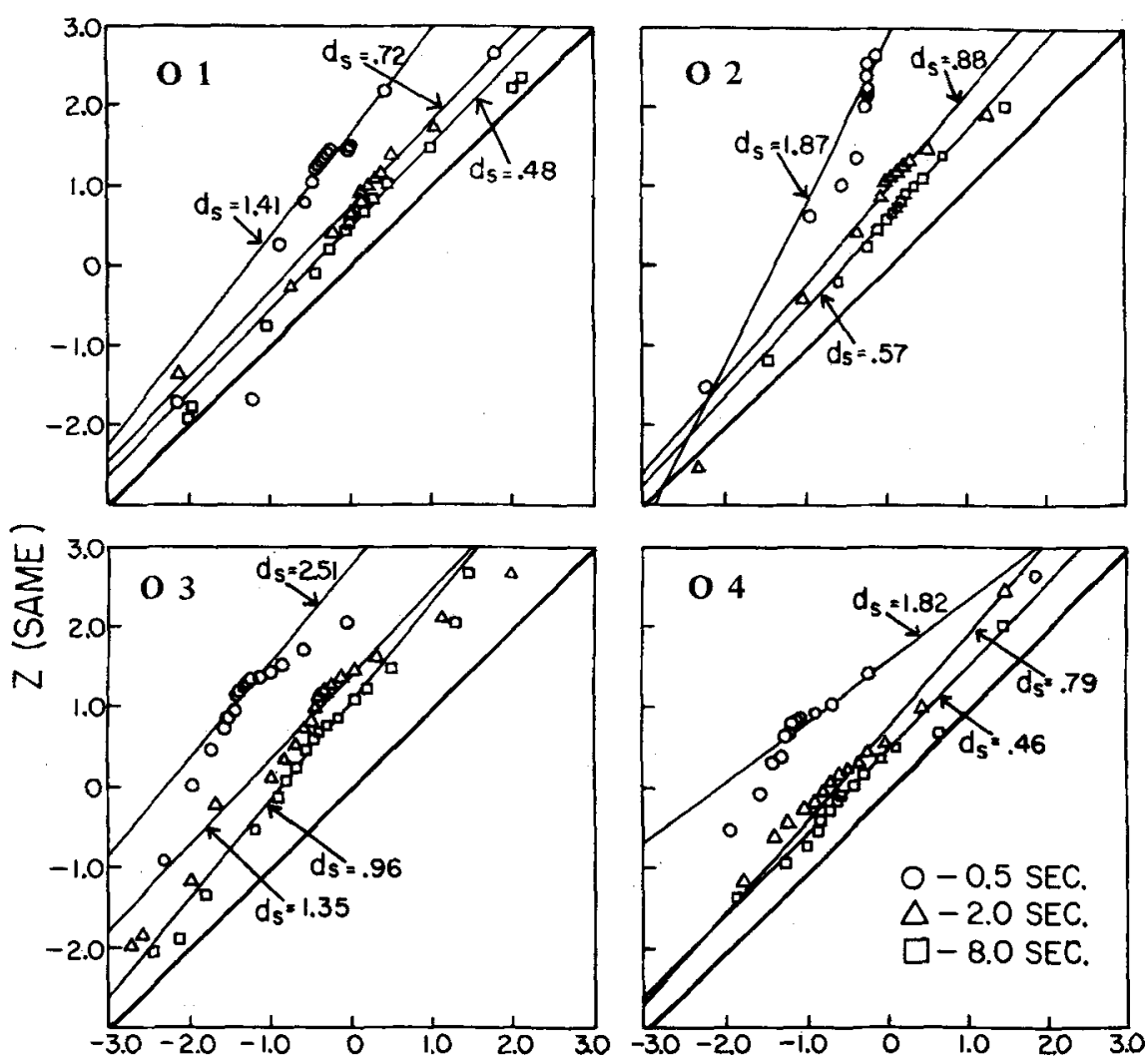

$Z$ (DIFFERENT) 
Table 4

Least Square Fits of Slopes (B) and Intercepts (A) for Rating and Latency ROC Functions

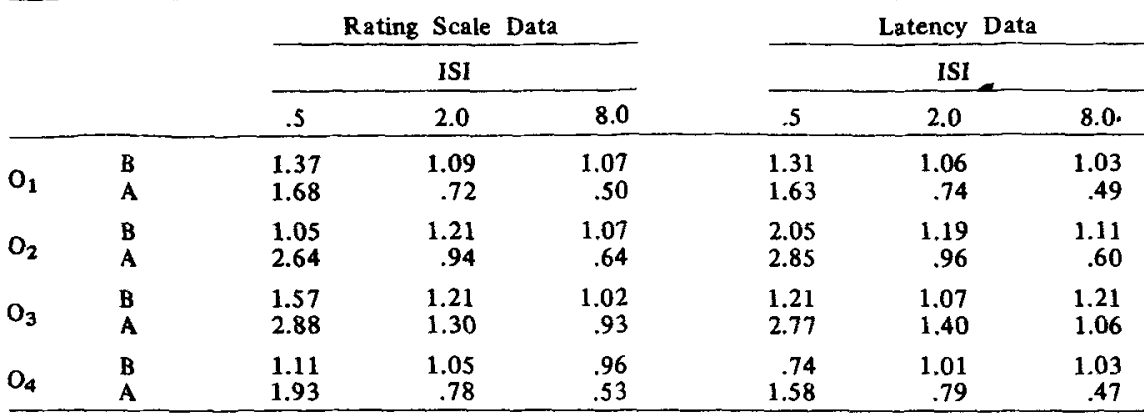

"yes-no" point, that pair of coordinates representing the overall proportion of "same" and "different" responses. The other criteria are variable, the result of having less variance at the "yes-no" point is the peak in the curve at that point. This hypothesis has difficulty in accounting tor the flattening of the peak with increased ISI.

A simpler explanation of the peaking phenomenon, including its reduction with increased ISI, lies in the nature of the distributions of response times. It is well known that response-time distributions tend to be markedly skewed with short times predominating. This is the case in the present study and was probably true also in the Norman and Wickelgren study. Furthermore, the lower categories of the 40-point "scale" will be most frequently represented on "same" trials (since these categories represent "same" responses), while the higher categories will be most frequently represented on "different" trials. The result will be cumulative probability distributions for same and different trials similar to those in Fig. 4. If this is the case, there will, of necessity, be a peak in the ROC curve. The peak is reduced under the longer delays simply because the latency distributions become less skewed; long latencies become relatively more frequent. The occasional peak noted by Wickelgren (1968) in ROC curves obtained from rating data presumably reflect unequal usage of the points on the rating scale.

Although the above argument does not negate Wickelgren's contention that the criteria vary about their respective means, it is apparent that the frequency of usage of various scale categories, whether rating or response time scales, do not necessarily reflect the underlying response strength distributions. Estimates of $d^{\prime}$ are probably affected only slightly, but inferences about slope and shape may be more sensitive to the scale. If precision in determining the ROC function is required, the application of any new category procedure should probably be validated by experimental manipulation of false-alarm rates. More generally, investigators might examine the distributions of category usage carefully and combine several end categories where this seems warranted.

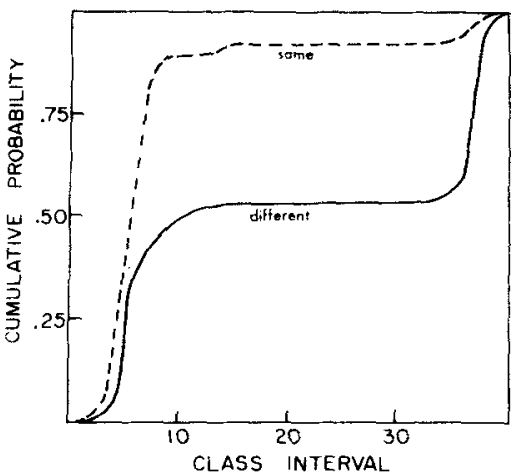

Fig. 4. Typical cumulative probability distributions of the latencies obtained from the present experiment. The peaks observed in the plotted data of Fig. 3 occur when cumulative probability distributions similar to those presented above are plotted against each other.
REFERENCES

AIKEN, E. G., \& LAU, A. W. Memory for the pitch of a tone. Perception \& Psychophysics, $1966,1,231-233$

BINDRA, D., DONDERI, D. C., \& NISHISATO, $S$. Decision latencies of "same" and "different" judgments. Perception \& Psychophysics, 1968, 3, 121-130.

BINDRA, D., WILLIAMS, J. A. \& WISE, J. S. Judgments of sameness and difference: Experiments on decision time. Science, 1965 , $150,1625-1627$.

COLTHEART, M., \& CURTHOYS, 1 . Short-term recognition memory for pitch: Effects of a priori probability on response times and error rates. Perception \& Psychophysics, 1968, 4, 85-89.

EGAN, J. P., \& ClaRKE, F. R. Psychophysics and signal detection. In J. B. Sidowski (Ed.), Experimental methods and instrumentation in prychology. New York: McGraw-Hill, 1966.

GREEN, D. M., \& SWETS, J. A. Signal detection theory and psychophysics New York: Wiley, 1966.

HARRIS, J. D. The decline of pitch discrimination with time. Journal of Experimental Psychology, 1952, 43, 96-99.

MASSARO, D. Retroactive interference in short-term recognition memory for pitch. Unpublished doctoral dissertation, University of Massachusetts, 1968.

MELTON, A. W. Implications of short-term memory for a general theory of memory. Journal of Verbal Learning \& Verbal Behavior, $1963,2,1-21$.

NORMAN, D. A., \& WICKELGREN, W. A. Strength theory of decision rules and latency in retrieval from short-term memory. Joumal of Mathematical Psychology, 1969, 6, 192-208.

SWETS, J. A., TANNER, W. P., JR., \& BIRDSALL, T. G. Decision processes in perception. Psychological Review, 1961, 68, 301-340.

WAUGH, N. C., \& NORMAN, D. A. Primary memory. Psychological Review, 1965, 72 89-104.

WICKELGREN, W. A. Consolidation and retroactive interference in short-term memory for pitch. Journal of Experimental Psychology, $1966,72,250-259$.

WICKELGREN, W. A. Associative strength theory of recognition memory for pitch. Journal of Mathematical Psychology, 1969, 6, 13-61.

\section{NOTES}

1. This research was supported by a faculty research grant from the University of Massachusetts Graduate School.

2. Address: Department of Psychology, University of Massachusetts, Amherst, Massachusetts 01002 .

(Accepted for publication October 15, 1969.) 